\title{
A multiplier theorem for the Hankel transform.
}

\author{
Rafał KAPELKO
}

\begin{abstract}
Riesz function technique is used to prove a multiplier theorem for the Hankel transform, analogous to the classical HörmanderMihlin multiplier theorem [6].
\end{abstract}

The celebrated Hörmander-Mihlin multiplier theorem [6] says that if a function $m$ on $R^{n}$ satisfies the following condition

$$
\sup _{R>0} R^{-n} \sum_{|l| \leq k_{0}} \int_{R<|x|<2 R}\left|R^{|| \mid} D^{l} m(x)\right|^{2} d x<\infty
$$

for some integer $k_{0}>\frac{n}{2}$ then the operator $T_{m}$ defined by $\left(T_{m} g\right)^{-}=m \hat{g}$ is bounded on every $L^{p}\left(R^{n}\right), 1<p<\infty$.

Restriction of the theorem to the set of radial functions on $R^{n}$ gives the multiplier theorem on spaces $L^{p}\left(R_{+}, x^{2 \alpha+1} d x\right), 1<p<\infty$ with $\alpha=\frac{n-2}{2}$. The ordinary Fourier transform on $R^{n}$ has to be replaced by the Hankel transform

$$
\widehat{f}(y)=2^{\alpha} \Gamma(\alpha+1) \int_{0}^{\infty} f(x)(y x)^{-\alpha} J_{\alpha}(x y) x^{2 \alpha+1} d x,
$$

where $J_{\alpha}$ is the Bessel function of the first kind of order $\alpha$.

The assumption (1) gets even the simpler form

$$
\sup _{R>0}\left(\int_{R}^{2 R}\left|x^{k} m^{(k)}(x)\right|^{2} \frac{1}{x} d x\right)^{\frac{1}{2}}<\infty,
$$

1991 Mathematics Subject Classification: 42B15, $42 \mathrm{C} 99$.

Servicio Publicaciones Univ. Complutense. Madrid, 1998. 
where $k=0,1,2, \ldots, k_{0}$ and $k_{0}>\alpha+1$.

It is quite natural to expect that the multiplier theorem should have an extension to all values $\alpha \geq \frac{1}{2}$ of the real parameter. However the exact repetition of the Hörmander proof does not lead to effect, mainly because the Hankel transform of the derivative of a function has no representation in terms of the transformation of the function. In order to omit this difficulty there were developed two technics in the literature.

The first one, [2], is indirect, uses a relation between the Jacobi polynomials and the Bessel functions but the result obtained there is weaker then expected. The proof goes under stronger assumption

$$
\sup _{R>0} R^{-1} \int_{R}^{2 R}\left|x^{k_{0}} m^{\left(k_{0}\right)}(x)\right|^{2} x^{-1} d x<\infty, k_{0}=[\alpha]+2 .
$$

The second one, [4], developes the original Hörmander's technique but instead of the ordinary derivative of a function it makes use of the powers of a Sturm-Liouville operator. The result is like the Hörmander one, but $k_{0}>\alpha+1$ must be an even number.

The aim of the note is to prove the multiplier theorem in full generality. We assume that $k_{0}$ is the least integer greater than $\alpha+1$. In fact $k_{0}$ may be a real number if one uses the Weyl fractional derivatives instead of ordinary derivatives. The main idea is based on the fact that the Hankel transform of Riesz function $R_{u}^{k_{0}}\left(x^{2}\right)$ has especially simple form. Then we follow the arguments of Gosselin and Stempak [4].

For a bounded function $m$ on $R_{+}$we define the multiplier operator $T_{m}$ by $\left(T_{m} g\right)^{-}=m \widehat{g}$, where denotes the Hankel transform (2).

Theorem 1. Fix $\alpha \geq \frac{1}{2}$ and let $k_{0}$ denote the least integer greater than $\alpha+1$. Assume that a bounded function $m$ on $R_{+}$satisfies

$$
\sup _{R>0}\left(\int_{R}^{2 R}\left|x^{k} m^{(k)}(x)\right|^{2} \frac{1}{x} d x\right)^{\frac{1}{2}}<\infty
$$

where $k=0,1, \ldots, k_{0}$. Then the operator $T_{m}$ is of weak-type $(1,1)$ and, consequently is bounded on every $L^{p}\left(R_{+}, x^{2 \alpha+1} d x\right), 1<p<\infty$.

In the proof we use the notion of the generalized convolution

$$
f * g(x)=\int_{0}^{\infty} f(y) T_{\alpha}^{y} g(x) y^{2 \alpha+1} d y
$$


where $T_{\alpha}^{y}$ is the generalized translation operator

$$
T_{\alpha}^{y} g(x)=b(\alpha) \int_{0}^{\pi} g\left((x, y)_{\theta}\right) \sin ^{2 \alpha}(\theta) d \theta,
$$

$(x, y)_{\theta}=\left(x^{2}+y^{2}-2 x y \cos \theta\right)^{\frac{1}{2}}, b(\alpha)=\pi^{-\frac{1}{2}} \Gamma(\alpha+1)\left(\Gamma\left(\alpha+\frac{1}{2}\right)\right)^{-1}$ and $f, g$ are suitable functions on the half-line (cf $[5]$ ).

As usual we use $C$ with subscripts or without subscripts for a constant which is not necessarily the same at each occurence.

Proof. The main idea of the proof is based on the fact that the Hankel transform of the function

$$
R(x)=\frac{1}{\Gamma\left(k_{0}\right)}\left(u-x^{2}\right)_{+}^{k_{0}-1}
$$

has a very simple form

$$
\hat{R}(x)=\Gamma(\alpha+1) 2^{\alpha+k_{0}-1}\left(\frac{\sqrt{u}}{x}\right)^{\alpha+k_{0}} J_{\alpha+k_{0}}(\sqrt{u} x) .
$$

(cf. [7, $\S 4$ Theorem 4.15]).

As usual we cut the function $m$ into small pieces by using a fixed bump function. Let $\Psi \in C_{0}^{\infty}\left(R_{+}\right)$with support in $(1,2)$ such that $\sum_{-\infty}^{\infty} \Psi\left(2^{-j} x\right)=1$ and $m_{j}(x)=m(x) \Psi\left(2^{-j} x\right)$. Define new family of functions $h(x)=m\left(x^{2}\right), h_{j}(x)=m_{j}\left(x^{2}\right)$. First using (3) and applying the method of [4], we will obtain the theorem for $h$. More precisisely we will prove

$$
\left\|T_{h} g\right\|_{p} \leq C_{1, p}\|g\|_{p} .
$$

Then we will show how to deduce the thesis for the function $m$ from the thesis for the function $h$.

For $h_{j}$ we write the reproducing formula

$$
h_{j}(x)=\frac{1}{\Gamma\left(k_{0}\right)} \int_{2^{j}}^{2^{j+1}} m_{j}^{\left(k_{0}\right)}(u)\left(u-x^{2}\right)_{+}^{k_{0}-1} d u
$$

By (3) we have

$$
\hat{h}_{j}(x)=\Gamma(\alpha+1) 2^{\alpha+k_{0}-1} \int_{2^{j}}^{2^{j+1}} m_{j}^{\left(k_{0}\right)}(u)\left(\frac{\sqrt{u}}{x}\right)^{\alpha+k_{0}} J_{\alpha+k_{0}}(\sqrt{u} x) d u .
$$


Then $T_{h}=\sum_{-\infty}^{\infty} T_{h_{j}}$ where $T_{h_{j}} g=\widehat{h}_{j} * g$ and $g \in L^{1}\left(R_{+}, x^{2 \alpha+1} d x\right)$. In order to prove (4) it is sufficient to establish (cf. $[4$, p.659] and $[1$, p.75]) that

$$
\sum_{j=-\infty}^{\infty} \int_{\left|x-y_{0}\right|>2\left|y-y_{0}\right|}\left|T_{\alpha}^{y \widehat{h}_{j}}(x)-T_{\alpha}^{y_{0}} \widehat{h}_{j}(x)\right| x^{2 \alpha+1} d x \leq C,
$$

with $C>0$ independent of $y, y_{0} 0$.

An application of Leibniz formula yields

$$
\left(\int_{2}^{2^{3+1}}\left|m_{j}^{\left(k_{0}\right)}(x)\right|^{2} d x\right)^{\frac{1}{2}} \leq C\left(2^{j}\right)^{\frac{1}{2}-k_{0}}
$$

where $C$ does not depend on $j$, and $k_{0}=\alpha+1+\epsilon$ for an $\epsilon>0$. We prove the following estimates:

$$
\begin{gathered}
\int_{t}^{\infty}\left|\widehat{h}_{j}(x)\right| x^{2 \alpha+1} d x \leq C\left(\sqrt{2^{j}} t\right)^{-\epsilon}, \\
\int_{0}^{\infty}\left|\widehat{h}_{j}(x)\right| x^{2 \alpha+1} d x \leq C .
\end{gathered}
$$

To prove (8) observe that by definition, $\hat{h}_{j}(x)$ coincides with the Hankel transform of the function

$$
I_{j}(y)=\frac{I^{\prime}(\alpha+1)}{\Gamma\left(\alpha+k_{0}+1\right)} \chi_{\left[\sqrt{2^{2}}, \sqrt{2^{j+1}}\right]}(y) m_{j}^{\left(k_{0}\right)}\left(y^{2}\right),
$$

with respect to the measure $d_{1} \mu(x)=x^{4 \alpha+3+2 \epsilon}$.

Now Schwartz' inequality, the Plancherel formula applied to $H_{j}$ and (7) give

$$
\begin{aligned}
\int_{t}^{\infty}\left|\hat{h}_{j}(x)\right| x^{2 \alpha+1} d x & \leq\left(\int_{0}^{\infty}\left|\widehat{h}_{j}(x)\right|^{2}\left(x^{2 \alpha+1+\frac{1}{2}+\epsilon}\right)^{2} d x\right)^{\frac{1}{2}}\left(\int_{t}^{\infty} \frac{1}{x^{1+2 \epsilon}} d x\right)^{\frac{1}{2}} \\
& =\left(\int_{0}^{\infty}\left|\widehat{h}_{j}(x)\right|^{2} x^{4 \alpha+3+2 \epsilon} d x\right)^{\frac{1}{2}} t^{-\epsilon} \frac{1}{\sqrt{2 \epsilon}} \\
& =C_{\alpha, k_{0}}\left(\int_{2^{j}}^{2^{j+1}}\left|m_{j}^{\left(k_{0}\right)}(p)\right|^{2} p^{2 \alpha+1+\varepsilon} d p\right)^{\frac{1}{2}} t^{-\epsilon} \frac{1}{\sqrt{2 \epsilon}} \\
& \leq C\left(2^{j}\right)^{\alpha+\frac{1}{2}+\frac{\varepsilon}{2}}\left(2^{j}\right)^{\frac{1}{2}-k_{0}} t^{-\epsilon}=C\left(\sqrt{2^{j}} t\right)^{-\epsilon} .
\end{aligned}
$$


To prove (9) we use (8). Now changing the variable $y=x \sqrt{u}$ in (5) we get

$$
\int_{0}^{2^{-\frac{j}{3}}}\left|\hat{h}_{j}(x)\right| x^{2 \alpha+1} d x \leq C_{3} \int_{2^{j}}^{2^{j+1}}\left|m_{j}^{\left(k_{0}\right)}(u)\right| u^{k_{0}-1} d u \int_{0}^{\sqrt{2}}\left|J_{\alpha+k_{0}}(y)\right| \frac{y^{2 \alpha+1}}{y^{\alpha+k_{0}}} d y .
$$

But Schwarz' inequality and (7) yield

$$
\int_{2^{j}}^{2^{j+1}}\left|m_{j}^{\left(k_{0}\right)}(u)\right| u^{k_{0}-1} d u \leq C_{1}\left(\int_{2^{j}}^{2^{j+1}}\left|m_{j}^{\left(k_{0}\right)}(u)\right|^{2} d u\right)^{\frac{1}{2}}\left(2^{j}\right)^{k_{0}-\frac{1}{2}} \leq C_{4} .
$$

Since $J_{\alpha+k_{0}}(x)$ is $x^{\alpha+k_{0}}$ asymptotically at $0^{+}$we have

$$
\int_{0}^{2^{-\frac{j}{2}}}\left|\widehat{h}_{j}(x)\right| x^{2 \alpha+1} d x \leq C_{2}
$$

Also, by (8)

$$
\int_{0}^{\infty}\left|\widehat{h}_{j}(x)\right| x^{2 \alpha+1} d x \leq C_{2}+\int_{2^{-\frac{j}{2}}}^{\infty}\left|\widehat{h}_{j}(x)\right| x^{2 \alpha+1} d x \leq C .
$$

Finally, to get (6) we use inequality (8) with estimates of Gosselin and Stempak (cf. [4, p.661])

$$
\begin{aligned}
\int_{\left|x-y_{0}\right| 2\left|y-y_{0}\right|} & \left|T_{\alpha}^{y} \widehat{h}_{j}(x)-T_{\alpha}^{y_{0}} \widehat{h}_{j}(x)\right| x^{2 \alpha+1} d x \\
& \leq \int_{\left|y-y_{0}\right|}^{\infty}\left|\widehat{h}_{j}(x)\right| x^{2 \alpha+1} d x+\int_{2\left|y-y_{0}\right|}^{\infty}\left|\widehat{h}_{j}(x)\right| x^{2 \alpha+1} d x \\
& \leq C_{1}\left(1+2^{-c}\right)\left(\sqrt{2^{j}}\left|y-y_{0}\right|\right)^{-\varepsilon}
\end{aligned}
$$

which will work for $\sqrt{2^{j}}\left|y-y_{0}\right| \geq 1$.

Since $h_{j}$ has support in $\left(0, \sqrt{2^{j+1}}\right)$ it follows from [4, Corollary 2.2] and (9) that

$$
\begin{aligned}
\int_{\left|x-y_{0}\right| 2\left|y-y_{0}\right|}\left|T_{\alpha}^{y} \widehat{h}_{j}(x)-T_{\alpha}^{y_{0}} \widehat{h}_{j}(x)\right| x^{2 \alpha+1} d x \\
\leq|| T_{\alpha}^{y} \widehat{h}_{j}-\left.T_{\alpha}^{y_{0}} \widehat{h}_{j}\right|_{L^{1}\left(R_{+}, x^{2 \alpha+1} d x\right)} \\
\leq C_{1} \sqrt{2^{j+1}}\left|y-y_{0}\right| \| \widehat{h}_{j}||_{L^{1}\left(R_{+}, x^{2 \alpha+1} d x\right)} \\
\leq \sqrt{2} C C_{1} \sqrt{2^{j}}\left|y-y_{0}\right|,
\end{aligned}
$$

which will be enough whenever $\sqrt{2^{j}}\left|y-y_{0}\right|<1$. 
This completes the proof of (6) and, consequently for the function $h$. The result for the function $m$ follows than from the lemma below.

Lemma 1. For $\alpha>0$ the transformation $x \rightarrow x^{\alpha}$ of $[0, \infty)$ induces the isomorphism $m(x) \rightarrow m\left(x^{\alpha}\right)$ of the space of all functions for which

$$
\|m\|_{2, k_{0}}=\sup _{R>0}\left(\int_{R}^{2 R}\left|x^{k} m^{(k)}(x)\right|^{2} \frac{1}{x} d x\right)^{\frac{1}{2}}<\infty
$$

for $k=0,1,2, \ldots, k_{0}$.

Proof. This is a simple consequence of fact that space $\|m\|_{2, k_{0}}$ is invariant under multiplication by $x^{\alpha}$ and Lebniz formula.

Remark. 'The method of Riesz function works when we use the Weyl fractional derivatives instead of ordinary derivatives.

A function $f$ on $R_{+}$has the Weyl fractional derivative of order $v>0$ if there exists a measurable function $g$ on $R_{+}$such that

$$
f(x)=\frac{1}{\Gamma(v)} \int_{x}^{\infty}(t-x)^{v-1} g(t) d l
$$

for almost all $x>0$. 'The function $g$ is unique up to a set of measure zero. It is denoted $f^{(v)}$ and called $v$-fractional derivative of order $v$.

The problem is that for a positive integer $v$ there exist smooth functions in the ordinary sense but not in the Weyl sense.

Theorem 2. Let $\mathrm{m}$ be a bounded function on $R_{+}$satisfies the condition

$$
\sup _{R>0}\left(\int_{R}^{2 R}\left|x^{v} m^{(v)}(x)\right|^{2} \frac{1}{x} d x\right)^{\frac{1}{2}}<\infty
$$

where $v>n+1, m^{(v)}$ is the Weyl fractional derivative. Then the operator $T_{n}$ is of weak-type $(1,1)$ and, consequently is bounded on every $L^{p}\left(R_{+}, x^{2 \alpha+1} d x\right), 1<p<\infty$.

Proof. As in the proof of Theorem 1 we define $h(x)=m\left(x^{2}\right)$ and obtain the theorem for function $h$. To do this we don't work with bump functions and define

$$
h_{j}(x)=\frac{1}{\Gamma(v)} \int_{2^{j}}^{2^{j+1}} m^{(v)}(u)\left(u-x^{2}\right)_{+}^{v-1} d u .
$$


Clerly $T_{h}=\sum_{-\infty}^{\infty} T_{h_{j}}$ where $T_{h} g=\widehat{h}_{j} * g$. The rest is the exact repetition of the proof of Theorem 1 . Finally the result for the function $m$ follows from lemma below.

Lemma 2. For $\alpha>0$ the transformation $x \rightarrow x^{\alpha}$ of $[0, \infty)$ induces the isomorphism $m(x) \rightarrow m\left(x^{\alpha}\right)$ of the space of all function for which

$$
\|m\|_{2, v}=\sup _{R>0}\left(\int_{\frac{R}{2}}^{R}\left|x^{\nu} m^{(v)}(x)\right|^{2} \frac{1}{x} d x\right)^{\frac{1}{2}}<\infty .
$$

Proof. The lenma is a modification of [3, Proposition 3.9]. The only difference is the norm $\|.\|_{(\mu), 2,1}$ is changed into the norm $\|\cdot\|_{2, v}$ and the proof is essentially the same.

\section{Acknowledgements.}

I would like to thank Tadeusz Pytlik who suggested me the method I am used and Krzysztof Stempak for useful suggestions, and fruitful discussions.

\section{References}

[1] R. Coifman and G.Weiss, Analyse harmonique non commutative sur certains espaces homogénes, Springer-Verlag, vol. 242, Berlin and New York, 1971.

[2] W. C. Connett and A. L. Schwartz, Weak type multipliers for Hankel transforms, Pac. Jour. of Math., vol.63, no. 1, 1976, 125-129.

[3] J. Galé and T.Pytlik, Functional calculus for infinitesimal generators of holomorphic semigroups, Jour. of Fun. Anal., vol.150, number 2, 1997, 307-355

[4] J. Gosselin and K. Stempak, A weak-type estimate for FourierBessel multipliers, Proc. of the Amer. Math. Soc., vol.106, no. 3, $1989,655-662$.

[5] I.Hirschman, Variation diminishing Hankel transforms, Jour. d'Anal. Math., vol.8, 1960/61, 307-336.

[6] L. Hörmander, Estimates for translation invariant operators in $L^{p}$ spaces, Acta Math., no. 104, 1960, 93-140. 
[7] E.M.Stein and G.Weiss, Introduction to Fourier analysis on Euclidean spaces, Princeton Univeristy Press, 1971.

Instytut Matematyczny

Uniwersytet Wrocławski

pl. Grunwaldzki $2 / 4$

50-384 Wroctaw

POLAND

Rafa! Kapelko

e-mail: kapelkoomath. uni.wroc.pl

Recibido: 8 de Diciembre de 1997

Revisado: 15 de Abril de 1998 